\title{
Major Crops Production Diseases/Pests Affecting them and their Controlling Methods in Debub Omo Zone
}

\author{
Kutoya Kusse ${ }^{1 *}$, Gedion Ermias ${ }^{1}$, Dawit Darcho ${ }^{1}$ and Muhaba Sultan ${ }^{2}$ \\ ${ }^{1}$ Ariba Minch University, Ethiopia \\ ${ }^{2}$ Southern Agricultural Research Institute, Ethiopia
}

Submission: September 07, 2018, Published: November 08, 2018

"Corresponding author: KutoyaKusse, Ariba Minch University, Jinka Agricultural Research Center Socio-economic researchers, Jinka, Ethiopia

\begin{abstract}
Agricultural production and productivity growth require fostering the linkages between the agricultural and nonagricultural sectors that is growth in agriculture does not occur independently of that in nonagricultural sectors. The production and productivity of the major crops in Debub Omo Zone is very low in relation to the land coverage due to poor agricultural technology utilization by the end users and low skill on farm management practices. To solve these problems and increase production and productivity, strong research extension system is important. The study was conducted in Debub Ari, Malle, Bena-Tsemay, Hammer and Dasenech Districts. The major crops grown in the area are Maize, Sorghum, Teff, Finger millet, Haricot bean, Ground net, Sesame, Sun flower, Sweet potato and cassava.
\end{abstract}

Keywords: Production; Productivity; Agricultural inputs; Production constraints

\section{Introduction}

Ethiopia is heavily reliant on agriculture as a main source of employment, income and foodsecurity for a vast majority of its population[1]. Agriculture generates $40 \%$ of gross domestic products (GDP)[2], and accounts for 85 and $90 \%$ of total employment and exports, respectively [1].Agriculture is the most important determinant of Southern Nation Nationality and peoples' Regional state ofEthiopia economy and it will continue toplayimportantroleinthe overalleconomydevelopmentoftheregion. However, an agricultural system in the region is at subsistence level.The livelihood ofover93\%ofthepeopleoftheregiondependonit, levelandfoodinsecurity problemisincreasingat shocking rate. Moreover, rapid natural resource degradation is prevalent [3].Also, Agricultural activities in the country as whole have been taking place under widely varying dynamic contexts such as physiographic, agro-ecology, climate and soil conditions. The success in the sector is then strongly influenced by topographic settings, degree ofhuman interferences and underlying biophysical features [4,5].

EconomyofEthiopialargelybasedonagriculturewhich accounts for $46.3 \%$ of the gross domestic product, $83.9 \%$ of exports, and $80 \%$ of the labor force in $2006 / 2007$, compared to $44.9 \%, 76.9 \%$ and $80 \%$ in 2002/2003, andagriculture remains the Ethiopian economy'smost importantsector [6].
Agricultureisa key factor inSouthern Nation Nationality and peoples Region economy and it will continuetoplaytheleadingroleintheoveralleconomic development of the region. Thefoodinsecurityproblemsintheareacausedby complex factors ranging from natural ones such as recurrent drought, degradation of natural resources, lack of appropriate technologies, weakinstitutional supportandlackof alternative employment [7]. Also farmingpracticesbroughtdisturbancestothe ecosystemsparticularly on soilsby disruptingthestable naturalbiogeochemicalprocessesofnutrientcycle,causing rapid nutrient depletion [8], and attributing to changes on the landscape characteristics $[9,10]$. This paper highlights the major crops production, challenges and controlling methods of farming system. Hence this study was conducted in farming system based six districts (Debub Ari, Malle, Bena-Tsemay, Hammer and Dasenech) of Debub Omo Zone with the following objectives:

a. To assess the area coverage production and productivity of major crops.

b. To identify the major crops production diseases and pests.

c. To differentiate the mechanisms to manage diseases and pests. 


\section{Methodology}

The study was conducted in Five Districts (Debub Ari, Malle, Bena-Tsemay, Hammer and Dasenech) of Debub Omo Zone. The districts were selected purposively based on crop production from eight districts found in the Zone. From these eight districts one agrarians from two agrarian districts and two pastoralist districts from four pastoralist districts were selected by probability sampling respectively, whereas the two semi-pastoralist districts were taken as they are purposively. The study was conducted by Socio-economic researchers of Jinka Agricultural research center. Before starting the study, the socio-economic researchers' team made short discussion on the preparation of checklist that helps to cover the study areas in accordance with the objective of the study.

A total of five districts were selected according to the aim of the study from those one agrarian, two pastoralist and two semi-pastoralist districts were covered. Based on the checklist in collaboration with each district Agricultural and natural resource management office data were collected from previously documented hard and soft copy materials with the help of crop extension and protection experts as well as from Zonal office from the year (2005-2010) on wards.

\section{Results and Discussion}

\section{Crop production and productivity}

Since agriculture constitutes a large share of national output and employs a majority of the labor force in most developing countries the sector has been the base for development. Agriculture has been seen as a low productivity, traditional sector that only passively contributed to development by providing food and employment. In the agriculture sector, major crops are the main source of food staple to most of people and provision of foreign exchange earnings and raw material to the industrial sector of the country as a whole and the zone in particular. It also enhances the food security of the society. According to these the major types of crops cultivated in the zone include cereals crops (maize, sorghum, Teff, Wheat, Barley, Finger millet) Pulses crops (Haricot bean, Field Pea, Mug bean) Oil Crops (Ground net, Sesame, Sun flower) and Root or Tuber Crops include (Sweet Potato, Potato, Cassava, Taro Inset). Farmer's whose livelihood depends on crop production produces the crops for consumption as well as for income generation and as a seed for further production. The type of cropping system they use in the zone is intercropping, sole cropping, crop rotation, fallowing. The area coverage of each type of crop, production and productivity recorded in the last five years from the year 2005-2009 E.C.

The table illustrates the major crops produced in the Zone according to their type, in which they are categorized as cereal crops, pulse crops, oil crops, root and tuber crops and vegetables. Also, the type of crop, name of crop, area in hectare, production and productivity of major crops in each district was identified. Based on this study words like area in hectare, production and productivity are explained below depending on the result of the study.

Area in (Ha): According to this study area in hectare shows the total amount of area covered by each major crop in each production year from 2005-2009 E.C for each district.

Production: Is the process of combining various inputs in order to get output. Based on this study production of the major crops indicate the output produced from the total amount of land in each five consecutive production year for each district by using inputs like chemicals, fertilizer seed land labor etc.

Productivity: Describes various measures of the efficiency of production. A productivity measure is expressed as the ratio of output to inputs used in a production process, i.e. output per unit of input. Following this principle, the study calculated productivity as; the total amount of output produced in each production year divided by the total amount of land used. Figuratively the data was arranged based on their importance in the table form below (Table 1).

\section{Input utilization to increase production and productiv- ity}

Agricultural inputs are defined as products permitted for use in production system. These include seed and fertilizer which used to increase the crops production and productivity.As indicate in the table above the agro pastoralists or agriculturalists in the study area uses the well-known inputs like seed and fertilizer in which some of the seeds they utilized were from government source while majority of the seed were from local or farmers themselves. Most of the time they used improved seed for cereal crops(Table 2).

\section{Major diseases and pests that affects production and productivity and the controlling methods}

Several insects/pests groups attack the flowers, fruits, leaves, steams, roots and branches of different crops. Diseases/ pests affecting the major type of cereal crops are MLD, armamo, cut worm, rust and wilt, pulse crops leaf miner, pod borer, pizzeria root rot, leaf spot, aphids, alternaria and tuta, oil crops mile bug, sting bug, green bug, cricket, fruit fly and root rot, Vegetables Root rot, Whitefly and Trips, powdery mildew, damping off, late blight, Root and tuber Crops, Moosic (virus) Leaf miner and wireworm, Powdery mildew, alternaria species, Mole rat, fungi and Cutworm whereas Fruits are affected by Powdery mildew, Trips (Pest), fruit fly and mango hoper(Table 3).

According to the table above producers undertake many activities to enhance production and productivity and to control diseases and pests that affect the crops they produce in their areas. Among the mechanisms they have used were using improved and disease resistant seed, increasing frequency of tillage, Crop rotation and shifting cultivation, Mechanical and chemical and that of cultural methods. 


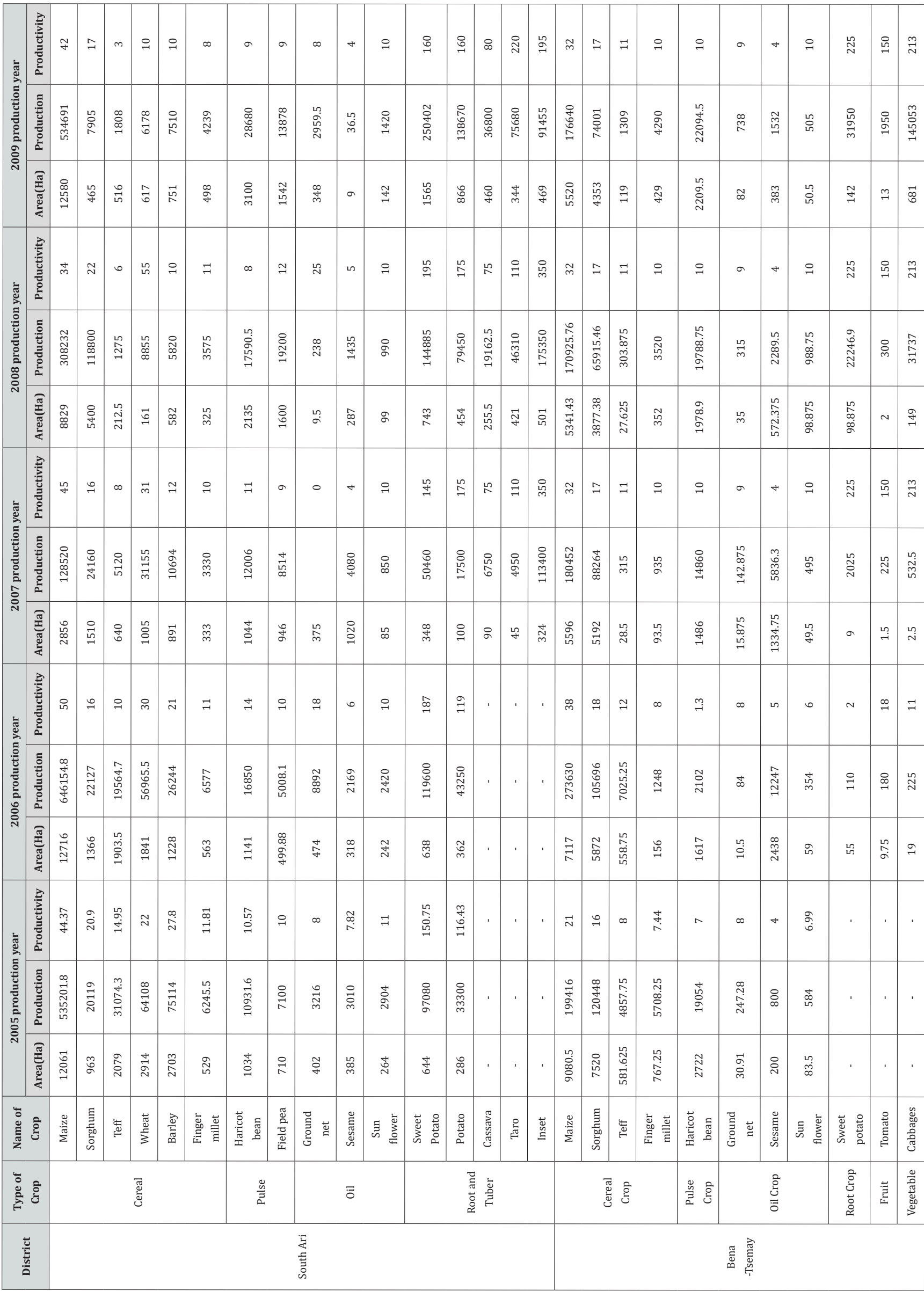


Agricultural Research \& Technology: Open Access Journal

\begin{tabular}{|c|c|c|c|c|c|c|c|c|c|c|c|c|c|c|c|c|}
\hline$\approx$ & N & + & & $\begin{array}{l}\stackrel{8}{0} \\
\stackrel{్}{1}\end{array}$ & $\stackrel{\infty}{\sigma}$ & N & 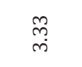 & 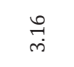 & 寍 & $\underset{\forall}{*}$ & 恚 & $\begin{array}{c}\text { Sे } \\
\text { s. }\end{array}$ & 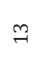 & $\exists$ & 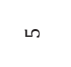 & $\stackrel{L}{m}$ \\
\hline 登 & 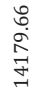 & 尽 & . & 总 & 怘 & 莕 & 吕 & $\stackrel{m}{\stackrel{m}{7}}$ & 嘿 & 范 & 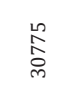 & 苦 & 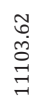 & 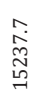 & 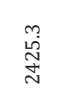 & 涵 \\
\hline 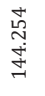 & 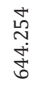 & 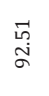 & & 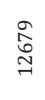 & 点 & 怘 & ్ָల్ & 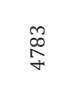 & $\stackrel{\infty}{\sim}$ & 节 & 知 & $\vec{~}$ & 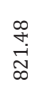 & 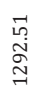 & $\begin{array}{l}\text { 芴 } \\
\text { ơ }\end{array}$ & జ్ \\
\hline$\approx$ & $\approx$ & m & & $\stackrel{m}{\text { N }}$ & \&. & & $\begin{array}{l}\vec{b} \\
\stackrel{\omega}{\circ}\end{array}$ & $\stackrel{\circ}{\not}$ & $\begin{array}{l}\infty \\
\substack{\infty \\
\infty}\end{array}$ & $\begin{array}{l}\vec{\omega} \\
\overrightarrow{+}\end{array}$ & $\stackrel{\text { II }}{\underset{J}{J}}$ & 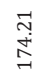 & $\stackrel{\infty}{\rightarrow}$ & $\stackrel{2}{2}$ & in & $\stackrel{\circ}{\stackrel{\Omega}{~}}$ \\
\hline \ָ & $\begin{array}{l}\text { Oे } \\
\stackrel{0}{0}\end{array}$ & 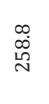 & . & 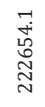 & 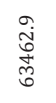 & . & 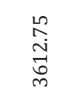 & 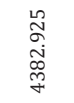 & 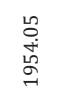 & 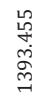 & 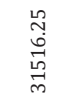 & 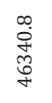 & $\begin{array}{l}n \\
\stackrel{n}{0} \\
\text { d. } \\
0\end{array}$ & $\begin{array}{l}\vec{i} \\
\text { d. } \\
\text { d. } \\
\text { d. }\end{array}$ & $\underset{⿱}{ \pm}$ & 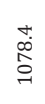 \\
\hline i & $\stackrel{\mathscr{\infty}}{\infty}$ & 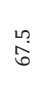 & & 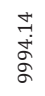 & 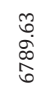 & & 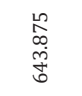 & 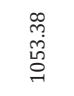 & 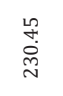 & 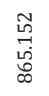 & 足 & 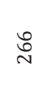 & 递 & 峁 & \begin{tabular}{l}
$\infty$ \\
市 \\
\multirow{j}{*}{}
\end{tabular} & 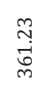 \\
\hline$\stackrel{\infty}{\sim}$ & $m$ & $\sim$ & + & $\begin{array}{c}\text { a. } \\
\text { a. }\end{array}$ & $\stackrel{\vec{\Phi}}{\stackrel{\vec{D}}{\sim}}$ & ' & iิ & 号 & 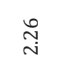 & 莒 & 喁 & $\infty$ & $\stackrel{\infty}{\sim}$ & $=$ & $\overrightarrow{\text { if }}$ & 第 \\
\hline 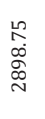 & 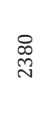 & $\begin{array}{l}\underbrace{2}_{0} \\
0 \\
0\end{array}$ & 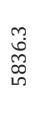 & 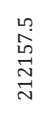 & 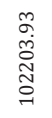 & . & $\begin{array}{l}\text { Na } \\
\stackrel{N}{N}\end{array}$ & 莣 & 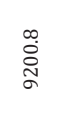 & 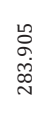 & 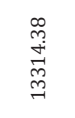 & $\underset{\infty}{\infty}$ & 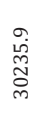 & 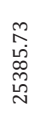 & 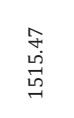 & $\begin{array}{l}\text { ț. } \\
\text { dू }\end{array}$ \\
\hline 惫 & 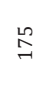 & సิ & 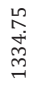 & 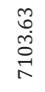 & 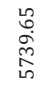 & & 兄 & $\begin{array}{l}\text { 芯 } \\
\stackrel{d}{0}\end{array}$ & 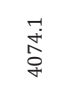 & 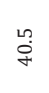 & 吕 & 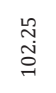 & 品 & 等 & 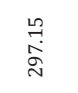 & 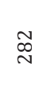 \\
\hline$\stackrel{8}{\sim}$ & $\stackrel{\infty}{\sim}$ & $\sigma$ & n & 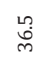 & $\begin{array}{l}\stackrel{g}{+} \\
\dot{+}\end{array}$ & ' & & 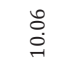 & 䀜 & $\underset{\dot{j}}{\hat{j}}$ & $\stackrel{\circ}{\circ}$ & స్ & $\approx$ & $m$ & 0 & 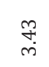 \\
\hline 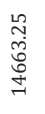 & 旡 & 足 & $\underset{\text { I }}{\mathbb{N}}$ & 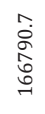 & 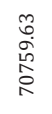 & . & & $\begin{array}{l}\text { 定 } \\
\text { 怘 } \\
\text { 品 }\end{array}$ & 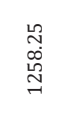 & 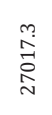 & 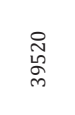 & 怘 & 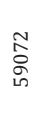 & 品 & 节 & 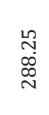 \\
\hline 总 & 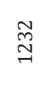 & 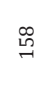 & 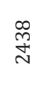 & 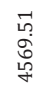 & 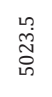 & & & 总 & 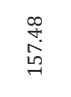 & 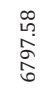 & \& & 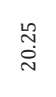 & 号 & 产 & $\bar{J}$ & $\stackrel{+}{+}$ \\
\hline$\hat{N}$ & $\stackrel{\text { N }}{\text { s. }}$ & + & + & $\overrightarrow{ల ్ p}$ & 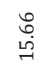 & $\Rightarrow$ & $\stackrel{\text { 모 }}{R}$ & $\underset{\infty}{\infty}$ & + & F & 8 & 熍 & \pm & 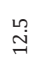 & $\underset{\substack{\mathscr{W} \\
+\infty}}{\mathscr{N}}$ & . \\
\hline 莺 & 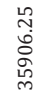 & ஜ̊ & ঃ & 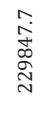 & 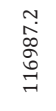 & 衤 & 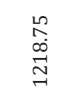 & 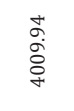 & 品 & 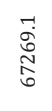 & 各 & 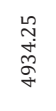 & \begin{tabular}{l} 
号 \\
\multirow{2}{*}{} \\
\multirow{\sigma}{*}{}
\end{tabular} & 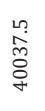 & 㖞 & \\
\hline 品 & స్తి & $\stackrel{\underline{\sigma}}{0}$ & $\stackrel{\text { ڤે }}{ }$ & $\begin{array}{l}\overrightarrow{0} \\
0.0 \\
0 \\
0\end{array}$ & 辛 & 它 & 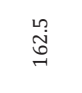 & 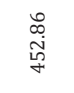 & $\begin{array}{c}\text { 心. } \\
\infty \\
\infty\end{array}$ & 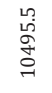 & $\stackrel{\vec{m}}{ }$ & 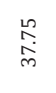 & 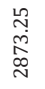 & :్లి & $\begin{array}{l}\text { 岂 } \\
\text { h }\end{array}$ & , \\
\hline$\frac{\mathscr{y}}{\tilde{m}}$ & 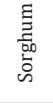 & 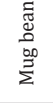 & 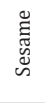 & 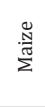 & $\begin{array}{l}\text { 䇏 } \\
\text { 音 } \\
\vdots\end{array}$ & 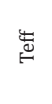 & 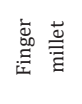 & 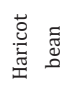 & 莺 & 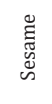 & 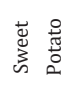 & 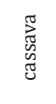 & $\frac{\tilde{s}}{2}$ & 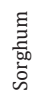 & 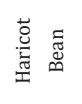 & 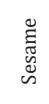 \\
\hline \multicolumn{2}{|c|}{ 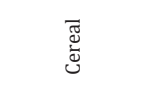 } & $\frac{\mathscr{0}}{\frac{g_{2}}{2}}$ & $\overline{0}$ & \multicolumn{4}{|c|}{ 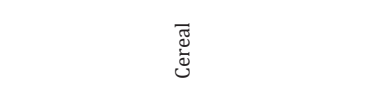 } & $\frac{\mathscr{m}}{2}$ & & & \multicolumn{2}{|c|}{ 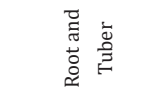 } & \multicolumn{2}{|c|}{ 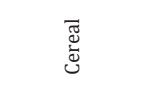 } & $\frac{\mathscr{m}}{\bar{z}}$ & $\overline{0}$ \\
\hline \multicolumn{4}{|c|}{ 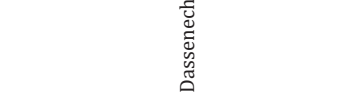 } & \multicolumn{9}{|c|}{ 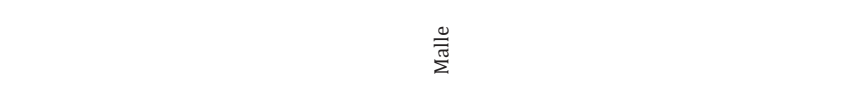 } & \multicolumn{4}{|c|}{ 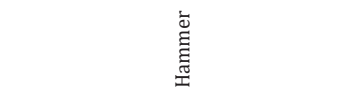 } \\
\hline
\end{tabular}


Table 2: Inputs used to increase production and productivity.

\begin{tabular}{|c|c|c|c|c|c|c|}
\hline \multirow{2}{*}{ Type of Crop } & \multirow{2}{*}{ Name of Crop } & \multirow{2}{*}{ Type of Seed } & \multirow{2}{*}{ Name of Variety } & \multirow{2}{*}{ Seed Source } & \multicolumn{2}{|c|}{ Fertilizer Used in (Kg) } \\
\hline & & & & & NPS & Urea \\
\hline \multirow{6}{*}{ Cereal Crops } & Maize & Improved & $\begin{array}{c}\text { BH140/MELKASA2 / } \\
\text { MELKASA4 }\end{array}$ & Gov't & 100 & 100 \\
\hline & Sorghum & improved & Gubiye and Abishire & Gov't & 100 & 100 \\
\hline & Teff & Improved & Kunicho & Gov't & 100 & 100 \\
\hline & Wheat & Local/Improved & Damip & Farmers/Gov't & 100 & 100 \\
\hline & Barley & Local & Local & Farmers & No & No \\
\hline & Finger millet & Local & Local & Farmers & No & No \\
\hline \multirow{3}{*}{ Pulse Crops } & Haricot bean & Improved & Nassir & Gov't & No & No \\
\hline & Mug bean & Local & Local & Farmers & No & No \\
\hline & Field Pea & Local & Local & Farmers & No & No \\
\hline \multirow{3}{*}{ Oil Crops } & sesame & Improved & Humera & Gov't & 100 & 50 \\
\hline & Sun flower & Local & Local & Farmers & No & No \\
\hline & Ground nut & Local & Local & Farmers & No & No \\
\hline Vegetables & Cabbages & Local & Local & Farmers & 50 & 50 \\
\hline \multirow{5}{*}{ Root and tuber } & Potato & Local/Improved & Local/Hawassa 84 & Farmers & No & No \\
\hline & Sweet potato & Local/Improved & Local & Farmers/Gov't & 50 & 50 \\
\hline & Cassava & Local & Local & Farmers & 50 & 50 \\
\hline & Taro & Local & Local & Farmers & 50 & 50 \\
\hline & Banana & Local & local & Farmers & No & No \\
\hline \multirow{3}{*}{ Fruits } & Papaya & Local & Local & Farmers & No & No \\
\hline & Avagado & Local & Local & Farmers & No & No \\
\hline & Mango & Local & Local & Farmers & No & No \\
\hline
\end{tabular}

Source: Woreda and Zonal Agriculture and Natural Resource Management Office.

Table 3: major type diseases/pests and their controlling methods.

\begin{tabular}{|c|c|c|c|}
\hline Type of crop & Name of crop & Diseases/pests affecting them & Controlling methods \\
\hline \multirow{6}{*}{ Cereal Crops } & Maize & $\begin{array}{l}\text { MLDandArmamo, cut worm, weeds and fall } \\
\text { army worms }\end{array}$ & Mechanical, chemical and cultural \\
\hline & Sorghum & Armamo & Mechanical and chemical \\
\hline & Teff & Rust & Cultural or chemical \\
\hline & Wheat & Wilt & Mechanical and chemical \\
\hline & Barley & Wilt & Mechanical and chemical \\
\hline & Finger millet & Rust & Crop rotation and shifting cultivation \\
\hline \multirow{2}{*}{ Pulse Crops } & Haricot bean & Leaf miner, Pod borer, Pizzeria and Root Rote & Mechanical and chemical \\
\hline & Mug bean & Leaf miner, Pod borer, Pizzeria, Root Root & Mechanical and chemical \\
\hline \multirow{3}{*}{ Oil Crops } & sesame & Mile Bug, Sting bug, Green bug andCricket & Mechanical and chemical \\
\hline & Sun flower & Fruitfly & Mechanical and chemical \\
\hline & Ground nut & Root rot & Mechanical and chemical \\
\hline Vegetables & Cabbages & $\begin{array}{l}\text { Root, rot, Whiteflyand Trips, powdery mildew } \\
\text { damping off, late blight }\end{array}$ & $\begin{array}{l}\text { Using improved disease resistant seed and Crop } \\
\text { rotation }\end{array}$ \\
\hline \multirow{5}{*}{ Root and tuber Crops } & Beet root & Moosic(virus) & Increasing frequency of tillage Crop rotation \\
\hline & Onion & Leaf miner and wireworm & Mechanical and chemical \\
\hline & Potato & Powdery mildew, alternaria species & Mechanical and chemical \\
\hline & Sweet potato & Mole rat and fungi & Crop rotation and shifting cultivation \\
\hline & Cassava & Cutworm & Crop rotation \\
\hline
\end{tabular}




\section{Agricultural Research \& Technology: Open Access Journal}

\begin{tabular}{|l|l|l|l|}
\hline \multirow{3}{*}{ Fruits } & Banana & Anthracnose(Fungi) & Mechanical and chemical \\
\cline { 2 - 4 } & Papaya & Powdery mildew & Mechanical and chemical \\
\cline { 2 - 4 } & Mango & Trips (Pest), fruit fly, mango hoper & Mechanical and chemical \\
\hline
\end{tabular}

Source: Woreda and Zonal Agriculture and Natural Resource Management Office

\section{Conclusion and recommendations}

Agriculture remains the key sector for food security, employment, growth, despite improper land use, agro-ecological zones, production and consumption patterns. Agriculture lead growth has the largest impact on reducing the depth of poverty. Thelivelihoodofover93\%ofthepeopleofSouthern Nation Nationalityand peoples Regionalstate ofEthiopia dependent on agriculture; however, agricultural system in the regionisatsubsistencelevel.Much effort has been needed to Produce or adapt agricultural technologies that would help to boost production and productivity but only few technologies adopt by the end users. There are a number of production problems which occurs at different section of production like input utilization (seed and fertilizer), land fragmentation pests and diseases. Producers in the study area do not uses production inputs in a recommended amount for all of the crops listed at a right time to enhance the production and productivity. To enhance production and productivity of the major crop's introduction of packages of modern inputs like improved seeds, fertilizers, pesticides and chemicals that dramatically increases crop production is important at recommended amount and right time to enhance food security. To address the packages to the small holder farmers, public interventions were crucial, so there should be strong relationship between research, extension, and the small land holding farmers involved for alleviating these production, and technologicalproblems. Finally, adoption of improved technologies which helps in production and marketing of these agricultural products are very essential in the studied area as a result all of the crops and others are recommended based on amount of land, market demand and other natural and manmade factors which affects productivity.

\section{References}

1. IFDC (International Fertilizer Development Center) (2012) Very essential in the studied area. Ethiopian fertilizer assessment. IFDC in support of African Fertilizer and Agribusiness Partnership. Alabama, USA, pp. 1-46.

2. UNDP (United Nations Development Programme) Ethiopia (2014) Quarterly economic brief: third quarter. Ethiopia, pp. 1-12.

3. Almekinders SJM, Elings A (2001) Collaboration, crop improvement in perspective. Euphytica 122(3): 425-438.

4. Chamberlin J, Emily S (2011) Ethiopian agriculture: a dynamic geographic perspective. Development Strategy and Governance Division, International Food Policy Research Institute, Ethiopia Strategy Support Program II, Ethiopia, pp. 1-29.

5. Diwediga B, Wala K, Folega F, Dourma M, Woegan YA, et al. (2015) Biophysical and anthropogenous determinants of landscape Patterns and degradation of plant communities in Mo Hilly Basin (Togo). Ecol Eng 85: 132-143.

6. IMF (2008) The Federal Republic of Ethiopia: Selected Issues. Series International Ministry Fund Country Report No. 08/259, USA; pp. 1-51.

7. Matous P, Y Tado, Dagne Mojo (2013) Role of extension and ethnoreligious network in acceptance of resource conserving agricultural resources among Ethiopian farmers. International journal of agricultural sustainability 11(4): 301-316.

8. Yengoh GT (2012) Determinants of yield differences in small- scale food crop farming systems in Cameroon. Agric Food Secure 1: 19.

9. Alemu B (2015) The effect of land use land cover change on land degradation in the highlands of Ethiopia. J Env Earth Sci 5(1): 1-12.

10. Gebreselassie Y, Anemut F, Aisu S (2015) The effects of land use types, management practices and slope classes on selected soil physicochemical properties in Zikre watershed, North-Western Ethiopia. Springer Open J Env Sys Res 4: 1-7.

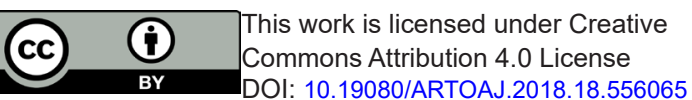

\section{Your next submission with Juniper Publishers will reach you the below assets}

- Quality Editorial service

- Swift Peer Review

- Reprints availability

- E-prints Service

- Manuscript Podcast for convenient understanding

- Global attainment for your research

- Manuscript accessibility in different formats

( Pdf, E-pub, Full Text, Audio)

- Unceasing customer service

Track the below URL for one-step submission https://juniperpublishers.com/online-submission.php 